\section{Calidad de vida, según percepción y comportamientos de control del peso por género, en estudiantes universitarios adolescentes en México}

\author{
Quality of life according to self-perceived weight, \\ weight control behaviors, and gender among \\ adolescent university students in Mexico
}

\author{
1 Universidad de \\ Guadalajara, Ciudad \\ Guzmán, México. \\ 2 Instituto Mexicano del \\ Seguro Social, Jocotepec, \\ México. \\ 3 Universidad de Chile, \\ Santiago, Chile. \\ 4 Universidad de Santiago de \\ Chile, Santiago, Chile. \\ Correspondencia \\ C. A. Hidalgo-Rasmussen \\ Universidad de Guadalajara. \\ Venezuela No. 28, Col. Las \\ Américas, Ciudad Guzmán, \\ Jalisco, 49026, México. \\ chidalgor@hotmail.com
}

\begin{abstract}
The aim of this study was to analyze quality of life (QoL) according to self-perceived weight and weight control behaviors, by gender. The sample consisted of 2,401 adolescent students (1719 years of age) enrolled from 2007 to 2009 at a Mexican university; $61.9 \%$ were women, $19.4 \%$ worked, and $99.2 \%$ were single. An online self-administered questionnaire was used that included the perceptual module of the YQOL-R and seven items on body weight, adapted from YRBS 2007. Results: $52 \%$ of women and $31.7 \%$ of men were attempting to lose weight. The highest QoL scores were in students who felt they were near the right weight, those who were attempting to maintain the same weight, and those who exercised. Lowest QoL was reported by those who considered themselves overweight, were trying to lose weight, were eating less, were skipping meals, or were using unsupervised dieting, vomiting, or laxatives. In women, QoL differed between those maintaining the same weight, gaining weight, and losing weight, while QoL in men only differed for those attempting to gain weight. The findings could be useful in educational processes, preventive programs, and assessment of interventions.
\end{abstract}

Quality of Life; Diet; Obesity; Adolescent
Carlos Alejandro Hidalgo-Rasmussen 1 Alfredo Hidalgo-San Martín 1 Bettylú Rasmussen-Cruz 2 Rosa Montaño-Espinoza 3,4

\section{Introducción}

La preocupación internacional por los problemas de nutrición en la adolescencia se ha incrementado, sobre todo en relación a la prevalencia de sobrepeso y a los comportamientos de riesgo para el control del peso ${ }^{1}$.

La obesidad en la adolescencia ha sido asociada a diversas patologías, además de tener consecuencias psicológicas y sociales negativas, y más probabilidades de llegar a ser adultos obesos 2 .

En México el sobrepeso y obesidad en adolescentes representan un problema de salud pública y éste se ha incrementado en todas las edades y regiones, especialmente en mujeres 3,4 . El peso corporal no sólo tiene influencia en el aspecto físico, sino en el subjetivo, asociándose con las áreas psicológica y social de la persona. Asílo han reportado estudios 5,6,7,8 que han encontrado el exceso de peso asociado con puntuaciones más bajas en calidad de vida (CV), o calidad de vida relacionada con la salud (CVRS), que los de peso normal, otros estudios, sin embargo, no encontraron asociaciones entre sobrepeso y CVRS ${ }^{9}$ y entre sobrepeso y el aspecto psicosocial 5 .

La Organización Mundial de la Salud (OMS) define la calidad de vida como "la percepción del individuo de su posición en la vida en el contexto de la cultura y sistema de valores en el cual él vive y en relación a sus objetivos, expectativas, estándares y preocupaciones" 10 (p. 153). La CV es 
multidimensional, subjetiva, considera aspectos negativos y positivos, y debe reflejar las normas culturales de bienestar objetivo 11,12, además de tener aspectos comunes a todos los individuos 13 . También define la CVRS como la calidad de vida del individuo, asociada a su bienestar físico, mental y social 8 .

Los instrumentos de medición de la CV y la CVRS se suelen dividir en genéricos y específicos. Los primeros se usan para población general y frecuentemente sana, mientras que los segundos hacen énfasis en el impacto de algún problema específico de salud ${ }^{14}$. La mayoría de ellos están formados por una serie de dominios o dimensiones que conforman un área de la conducta que se pretende medir ${ }^{15}$ y siguen el modelo psicométrico.

El género parece ser importante para comprender cómo se asocia el peso a la CV o CVRS 5,7. En Estados Unidos de Norteamérica (EEUU) se observó que, con frecuencia, los adolescentes valoraban su peso corporal como inadecuado, a pesar de ser correcto 16 y con frecuencia ante su peso - real o subjetivo - intentaban modificarlo, en especial perderlo, sobre todo en mujeres 1. Ellas se preocupaban más del peso corporal y forma 17 y se comprometían más con comportamientos de control del peso que los hombres, probablemente, debido al énfasis de la sociedad occidental en la delgadez como ideal para las mujeres 18 . Estudios recientes también han reportado un incremento en la preocupación de los hombres adolescentes respecto al peso 17 .

Los comportamientos saludables de control del peso, como el ejercicio, se han asociado a una mayor CV 19 y satisfacción con la vida 9,20, mientras que los comportamientos de control del peso no saludable, en personas con desórdenes alimentarios, se han asociado a una menor CV o CVRS 21,22.

En México no hemos encontrado estudios relativos a las asociaciones sobre calidad de vida y percepción del peso, lo que intentan los adolescentes hacer con él y los comportamientos de control que llevan a cabo.

A partir de estos antecedentes, nuestro objetivo fue analizar la asociación de la auto percepción del peso y los comportamientos de control del peso y la calidad de vida, según género, en estudiantes universitarios en Jalisco, México.

\section{Métodos}

Estudio observacional, transversal y analítico, dirigido a estudiantes provenientes de Ciudad Guzmán, una ciudad considerada media en Jalisco por su desarrollo socioeconómico (100 mil habitantes aprox.), de algunas ciudades pequeñas (15 mil a menos de 100 mil habitantes) y de localidades mixtas y rurales (menores de 15 mil habitantes) de Jalisco, México. Contempló como marco de muestra el número de estudiantes que se matricularon en los periodos semestrales de los años 2007, 2008 y 2009 en el Centro Universitario del Sur de la Universidad de Guadalajara con un total de 4.472 alumnos para permitir identificar comportamientos de control del peso de prevalencia pequeña.

El cuestionario fue auto aplicado en línea a todos los estudiantes que se presentaron la primera semana de clases de cada semestre (3.114) e incluyó a los adolescentes de 17 a 19 años que aceptaron participar y que completaron el cuestionario en su totalidad (2.401). La muestra quedó caracterizada por un $61,9 \%$ de mujeres, $80,6 \%$ que no trabajaban y el $99,2 \%$ solteros o solteras.

Se recogió información de CV mediante el cuestionario Youth Quality of Life Instrument Research versión (YQOL-R) 23,24, en su versión en español de 61 reactivos, seleccionándose los ítems perceptuales que son aquellos conocidos sólo por el adolescente y que son los primarios para valorar la CV, utilizando la definición de CV de la OMS: "la percepción de su posición en la vida" 24 (p. 7). El instrumento está formado por cuatro dominios: el general, de 3 ítems, con declaraciones sobre la satisfacción con su vida y el valor de ésta y sentimiento de seguridad en la escuela; el personal, de 14 ítems, aborda los sentimientos acerca de sí mismo, respecto al estrés, la soledad, sentimientos y creencias, las dificultades y errores, la exclusión, la energía y sexualidad; el relacional, con 14 ítems, acerca de las relaciones con la familia, adultos, amigos, pares y satisfacción con su vida social y por último el ambiental, con 10 ítems sobre oportunidades y obstáculos para el futuro, la educación y acceso a la información, el dinero, el disfrutar lo nuevo y seguridad en el hogar. Se usó para cada ítem una escala de Likert de 11 opciones. Los índices se crearon llevando las puntuaciones de los ítems a puntuación T, para reflejarse en una escala de CV de 0 a 100 (donde la mayor puntuación significa calidad de vida superior), obteniéndose a partir de allí medias y desviación estándar, así como percentiles con sus puntos de corte. El instrumento ha sido validado para 236 adolescentes norteamericanos de 12 a 18 años con y sin discapacidad, la validez de constructo con el KINDL Children Quality of Life Questionnaire 25, obtuvo una correlación de 0,73 , indicando significativa asociación entre las dos medidas, la consistencia interna mediante alfa de Cronbach 0,77 a 0,96 y la reproducibilidad con el coeficiente de correlación intra clase 0,74 a 0,85 24,26. La versión en español del instrumento 
fue adaptada también para Puerto Rico en dos grupos méxico-americanos y portorriqueños 27 . Al ser aplicado a la población de nuestro estudio, su consistencia interna superó el estándar 28 para resultados grupales en el índice total $(0,92)$ y sus dominios: general $(0,86)$, personal $(0,81)$, relacional $(0,85)$ y ambiental $(0,76)$.

El cuestionario indagó sobre percepción y comportamientos de control del peso mediante siete ítems adaptados del 2007 YRBS: Youth Risk Behavior Survey del National Center for Chronic Disease Prevention and Health Promotion (NCCDPHP) 29. Consideramos a Katchadourian ${ }^{30}$ en su concepción de comportamiento, como aquella actividad de un individuo, incluyendo los fenómenos observables e informes verbales acerca de estados o experiencias subjetivas. Las opciones de respuesta fueron categóricas y su nivel de medición nominal u ordinal. Las primeras dos preguntas indagaban sobre la percepción de su peso y qué había tratado de hacer con éste (mantenerlo, subirlo, bajarlo o no hacer nada), mientras que las otras cinco preguntaban si en los últimos 30 días había llevado a cabo comportamientos de control del peso (hacer ejercicio, comer menos alimentos, menos calorías o comidas bajas en grasa dejar de comer por 24 horas, hacer dieta de píldoras o polvos sin supervisión médica, vomitar o tomar laxantes) para mantenerlo o bajarlo.

\section{Ética}

El proyecto fue aprobado por las autoridades universitarias, después de analizarlo a la luz de los postulados de la Declaración de Helsinki de las Pautas Éticas Internacionales para la Investigación Biomédica en Seres Humanos (1964, reformulada 2000), de la Asociación Médica Mundial. Esta investigación se consideró de "riesgo mínimo", de acuerdo con la Ley General de Salud de México. A los alumnos se les dio a conocer el proyecto antes de responder el cuestionario. La participación fue voluntaria y con consentimiento informado. Los datos fueron anónimos y confidenciales.

\section{Estadística}

Después de los procedimientos estadísticos descriptivos se realizaron pruebas estadísticas para la comparación de medias. En el caso de las variables categóricas se usó la prueba de chi cuadrada, y en las con dos medias se usó prueba $t$ para muestras independientes. Para las variables con más de dos medias se usó ANOVA cuando ésta resultó significativa, se utilizaron como pruebas post hoc las comparaciones múltiples:
T2 de Tamhane, cuando no se asumió la homogeneidad de varianzas, y mediante el estadístico de Levene y Scheffé, cuando sí se asumió. En todos los análisis se adoptó un nivel de significación del $5 \%$. Se obtuvieron para los índices de calidad de vida, medias, desviaciones estándar y se determinaron percentiles. Se usó como puntos de corte los percentiles (P) 33 y 66 para generar valores de CV inferiores (menos de P33), medios (entre P33 y menos P66) y superiores (de P66 en adelante). En los análisis se utilizó el software estadístico SPSS V17 (SPSS Inc., Chicago, EEUU) y Stata 10.0 (Stata Corp., College Station, EEUU).

\section{Resultados}

No hubo asociación de género con estado civil, origen campesino o no, trabajo del padre, ni con año de ingreso, mientras que sí fue significativa por edad (mayor frecuencia para las mujeres en edades menores) y trabajo (los hombres con mayor frecuencia de trabajo).

Más de la mitad de los participantes percibieron su peso cercano al correcto. Reportaron sobrepeso $36,5 \%$ de las mujeres y $30,6 \%$ de los hombres. Casi la mitad de la población trataba de bajar de peso. Los comportamientos más practicados para bajar de peso fueron hacer ejercicio y dejar de comer. Todos los comportamientos de control del peso tuvieron una prevalencia mayor al $1 \%$. Se asoció significativamente el género a percepción del peso, a lo que ha tratado de hacer con su peso y a comportamientos de control del peso, excepto hacer ejercicio y dejar de comer durante 24 horas (Tabla 1).

Los índices de calidad de vida fueron mayores a 80 puntos. En la población total, el dominio con mayor puntuación media fue el general y el que tuvo la menor fue el personal. Las puntuaciones del dominio personal fueron significativamente más altas en hombres que en mujeres. Los valores inferiores del índice total de CV se ubican bajo 86,6 (P33) y los valores superiores a partir de 92,5 (P66) (Tabla 2).

Los participantes que reportaron peso cercano al correcto tuvieron la puntuación más alta en CV y los que reportaron mucho sobrepeso la más baja para la población total y por género. Para el índice total en las mujeres hubo diferencias significativas entre percepción de peso correcto y todas las categorías, excepto ligero sobrepeso, en cambio los hombres sólo tenían diferencias con esa categoría. En cuanto a los dominios en población total y mujeres hubo diferencias entre percepción de peso correcto y sobrepeso, no así en hombres con peso correcto, que tenían diferencias en el dominio personal 
Percepción y comportamientos de control del peso en población total, y por género, en adolescentes mexicanos.

\begin{tabular}{|c|c|c|c|c|c|}
\hline & \multicolumn{2}{|c|}{$\begin{array}{l}\text { Población total } \\
\qquad(n=2.401)\end{array}$} & \multirow{2}{*}{$\begin{array}{c}\text { Mujeres } \\
(n=1.486) \\
\%\end{array}$} & \multirow{2}{*}{$\begin{array}{c}\text { Hombres } \\
(n=915) \\
\%\end{array}$} & \multirow[t]{2}{*}{ Valor-p * } \\
\hline & $\mathrm{n}$ & $\%$ & & & \\
\hline \multicolumn{6}{|l|}{ Percepción del peso } \\
\hline Muy bajo de peso & 32 & 1,3 & 1,1 & 1,7 & 0,002 \\
\hline Ligero bajo de peso & 166 & 6,9 & 5,9 & 8,6 & \\
\hline Cerca del peso correcto & 1.381 & 57,5 & 56,6 & 59,0 & \\
\hline Ligero sobrepeso & 726 & 30,2 & 31,8 & 27,8 & \\
\hline Con mucho sobrepeso & 96 & 4,0 & 4,7 & 2,8 & \\
\hline \multicolumn{6}{|l|}{ Qué trata de hacer con su peso } \\
\hline Bajar de peso & 1.074 & 44,7 & 52,8 & 31,7 & 0,000 \\
\hline Mantenerme en el peso & 659 & 27,4 & 25,9 & 29,9 & \\
\hline No hacer nada con mi peso & 383 & 16,0 & 13,5 & 19,9 & \\
\hline Subir de peso & 285 & 11,9 & 7,8 & 18,5 & \\
\hline \multicolumn{6}{|l|}{ Hacer ejercicio } \\
\hline Sí & 1.244 & 51,8 & 52,2 & 51,3 & 0,669 \\
\hline No & 1.157 & 48,2 & 47,8 & 48,7 & \\
\hline \multicolumn{6}{|l|}{ Comer menos } \\
\hline Sí & 877 & 36,5 & 42,5 & 26,8 & 0,000 \\
\hline No & 1.524 & 63,5 & 57,5 & 73,2 & \\
\hline \multicolumn{6}{|l|}{ Dejar de comer por 24 horas } \\
\hline Sí & 85 & 3,5 & 4,1 & 2,6 & 0,056 \\
\hline No & 2.316 & 96,5 & 95,9 & 97,4 & \\
\hline \multicolumn{6}{|l|}{ Dieta sin supervisión } \\
\hline Sí & 80 & 3,3 & 4,4 & 1,6 & 0,000 \\
\hline No & 2.321 & 96,7 & 95,6 & 98,4 & \\
\hline \multicolumn{6}{|l|}{ Vomitar/Laxantes } \\
\hline Sí & 60 & 2,50 & 3,2 & 1,3 & 0,003 \\
\hline No & 2.341 & 97,5 & 96,8 & 98,7 & \\
\hline
\end{tabular}

* El valor-p de comparación de géneros se obtuvo con chi cuadrada.

Tabla 2

Índices de calidad de vida total y por dominio, según población total y género, en adolescentes mexicanos.

\begin{tabular}{|c|c|c|c|c|c|c|c|c|c|}
\hline \multirow[t]{2}{*}{ Calidad de vida } & \multicolumn{4}{|c|}{$\begin{array}{l}\text { Población total } \\
\qquad(n=2.401)\end{array}$} & \multicolumn{2}{|c|}{$\begin{array}{c}\text { Mujeres } \\
(n=1.486)\end{array}$} & \multicolumn{2}{|c|}{$\begin{array}{l}\text { Hombres } \\
(n=915)\end{array}$} & \multirow[t]{2}{*}{ Valor-p * } \\
\hline & Media & DS & P33 & P66 & Media & DS & Media & DS & \\
\hline Índice total & 87,5 & 9,3 & 86,6 & 92,5 & 87,3 & 9,5 & 87,9 & 8,8 & 0,089 \\
\hline Dominio general & 91,7 & 12,3 & 90,0 & 100,0 & 91,4 & 12,3 & 92,1 & 12,3 & 0,157 \\
\hline Dominio personal & 83,0 & 11,3 & 80,7 & 89,3 & 82,3 & 11,7 & 84,0 & 10,5 & $<0,001$ \\
\hline Dominio relacional & 86,5 & 10,9 & 85,0 & 92,1 & 86,3 & 11,5 & 86,7 & 9,7 & 0,276 \\
\hline Dominio ambiental & 89,0 & 8,3 & 88,0 & 94,0 & 89,1 & 8,4 & 88,9 & 8,29 & 0,452 \\
\hline
\end{tabular}

DS: desviación estándar; P: percentil.

* Para obtener el valor-p para los géneros se utilizó la prueba t para muestras independientes. 
con sobrepeso y el relacional con mucho sobrepeso (Tabla 3 ).

En la Tabla 4 se observa que la CV total fue más alta para quienes tratan de mantener su peso que quienes tratan de bajar o subir de peso, tanto para la población total como para el grupo de mujeres, pero en los hombres solamente hay diferencias con bajar. Cuando hicimos estas comparaciones con la clasificación de superior, media e inferior de $\mathrm{CV}$, encontramos lo mismo. En cuanto a dominios tuvieron igual comportamiento, la CV fue más alta en quienes tratan de mantener su peso que quienes tratan de bajar de peso, excepto en hombres en el dominio ambiental. En mujeres la CV total fue más alta para aquellas que tratan de mantener su peso, respecto a las que quieren subir, excepto en el dominio relacional.

En cuanto a CV y comportamientos de control del peso (Tabla 5) se observó en la población total, que al comparar al grupo que llevó a cabo los comportamientos con los que no lo hicieron, la CV más alta fue para quienes hicieron ejercicio y más baja para quienes comieron menos, dejaron de comer 24 horas, hicieron dieta sin supervisión, vomitaron o tomaron laxantes para controlar su peso. Al separar por género, los resultados se repitieron para las mujeres, pero en los hombres no hubo diferencias significativas en la CV entre los que comieron menos, hicieron dieta sin supervisión, vomitaron o tomaron laxantes y los que no lo hicieron.

\section{Discusión}

En nuestro estudio el $34,2 \%$ de los estudiantes se perciben con sobrepeso, superior al $29,3 \%$ reportado por el YRBSS 2007 en estudiantes norteamericanos de grados 9 al 121 , y algo menor al $35,5 \%$ reportado por la Encuesta Nacional de Salud y Nutrición 2006 (ENSANUT) en población jalisciense de 12 a 19 años con medición objetiva 31. En el grupo con sobrepeso, por género, las

Índices de calidad de vida total y por dominios en población total y por género, según percepción del peso en adolescentes mexicanos.

\begin{tabular}{|c|c|c|c|c|c|c|c|c|c|c|}
\hline \multirow[t]{3}{*}{ Grupos/Calidad de vida } & \multicolumn{10}{|c|}{ Percepción del peso } \\
\hline & \multicolumn{2}{|c|}{ Muy bajo de peso } & \multicolumn{2}{|c|}{ Ligero bajo peso } & \multicolumn{2}{|c|}{$\begin{array}{c}\text { Cerca del peso } \\
\text { correcto }\end{array}$} & \multicolumn{2}{|c|}{ Ligero sobrepeso } & \multicolumn{2}{|c|}{ Mucho sobrepeso } \\
\hline & Media & DS & Media & DS & Media & DS & Media & DS & Media & DS \\
\hline \multicolumn{11}{|l|}{ Total $(n=2.401)$} \\
\hline Índice total & $82,8_{c}$ & 9,2 & $87,0_{d}$ & 9,2 & $88,9 \mathrm{abc}$ & 8,1 & $86,4_{b, e}$ & 9,7 & $79,3_{a, d, e}$ & 14,5 \\
\hline Dominio general & 87,1 & 15,5 & $90,9_{c}$ & 12,6 & $93,1_{a, b}$ & 11,0 & $90,8_{b, d}$ & 12,7 & $80,5_{a, c, d}$ & 21,1 \\
\hline Dominio personal & $74,3_{c, f, g}$ & 11,7 & $82,6_{d, f}$ & 11,3 & $84,9 a, b, c$ & 10,0 & $81,2_{b, e, g}$ & 11,7 & $72,7_{a, d, e}$ & 15,2 \\
\hline Dominio relacional & 83,3 & 9,7 & $86,1_{c}$ & 10,9 & $87,6_{a, b}$ & 9,8 & $85,4_{b, d}$ & 11,5 & $79,8_{a, c, d}$ & 16,3 \\
\hline Dominio ambiental & 86,6 & 7,1 & $88,4_{c}$ & 8,4 & $89,9 a, b$ & 7,6 & $88,2_{b, d}$ & 8,7 & $84,0_{a, c, d}$ & 12,0 \\
\hline \multicolumn{11}{|l|}{ Mujeres $(n=1.486)$} \\
\hline Índice total & $83,1_{c}$ & 5,4 & $86,4_{b, d}$ & 10,2 & $88,8_{a, b, c}$ & 8,1 & $86,0_{e}$ & 10,0 & $79,0_{a, d, e}$ & 14,4 \\
\hline Dominio general & $90,6 e$ & 8,5 & $89,6_{c}$ & 14,0 & $93,1_{a, b}$ & 10,0 & $90,3_{b, d}$ & 13,2 & $80,4_{a, c, d, e}$ & 20,8 \\
\hline Dominio personal & $71,9_{c, f}$ & 10,7 & $81,2_{d, f}$ & 12,1 & $84,5_{a, b, c}$ & 10,0 & $80,5_{b, e}$ & 12,1 & $71,9_{a, d, e}$ & 14,9 \\
\hline Dominio relacional & 83,8 & 8,0 & 86,0 & 11,9 & $87,6_{a, b}$ & 10,0 & $85,1_{b}$ & 12,2 & $79,3_{a}$ & 17,5 \\
\hline Dominio ambiental & 86,0 & 5,8 & 88,7 & 9,6 & $90,1_{a, b}$ & 7,6 & $88,2_{b}$ & 8,7 & $84,6 a$ & 11,0 \\
\hline \multicolumn{11}{|l|}{ Hombres $(n=915)$} \\
\hline Índice total & 82,5 & 12,1 & 87,7 & 8,0 & $88,9 a$ & 8,1 & $87,0_{a}$ & 9,0 & 79,8 & 15,2 \\
\hline Dominio general & 83,5 & 19,9 & 92,4 & 10,7 & 93,2 & 12,0 & 91,6 & 11,6 & 80,5 & 22,4 \\
\hline Dominio personal & 76,6 & 12,4 & 84,1 & 10,1 & $85,4_{a, b}$ & 9,5 & $82,5_{b}$ & 10,9 & $75,0_{a}$ & 16,3 \\
\hline Dominio relacional * & 82,7 & 11,4 & 86,2 & 9,8 & $87,6 a$ & 9,2 & 85,8 & 10,1 & $81,3_{a}$ & 12,7 \\
\hline Dominio ambiental & 87,1 & 8,4 & 88,2 & 7,0 & 89,6 & 7,7 & 88,3 & 8,8 & 82,5 & 14,3 \\
\hline
\end{tabular}

DS: desviación estándar.

Nota: la ANOVA de todos los grupos fue significativa con $p<0,01$. Para todos los grupos la prueba post hoc aplicada fue Tamhane's, excepto para aquel señalado con *, en el que la prueba post hoc aplicada fue Scheffé. En cada fila las medias que comparten algún subíndice (letras a, b, c, d, e) son significativamente diferentes entre ellas con una $p<0,05$. 
Índices de calidad de vida total y por dominios en población total y por género, según lo que trata de hacer con su peso en adolescentes mexicanos.

\begin{tabular}{|c|c|c|c|c|c|c|c|c|}
\hline \multirow[t]{2}{*}{ Grupo/Calidad de vida } & \multicolumn{2}{|c|}{ Bajar de peso } & \multicolumn{2}{|c|}{ Mantener el peso } & \multicolumn{2}{|c|}{ No hacer nada } & \multicolumn{2}{|c|}{ Subir de peso } \\
\hline & Media & DS & Media & DS & Media & DS & Media & DS \\
\hline \multicolumn{9}{|l|}{ Total $(n=2.401)$} \\
\hline Índice total & $86,5_{a}$ & 10,0 & $89,4 a, b$ & 7,5 & 87,8 & 9,3 & $86,8_{b}$ & 9,4 \\
\hline Dominio general & $90,5_{a}$ & 10,0 & $94,1_{a, b, c}$ & 8,4 & $91,7_{b}$ & 12,5 & $90,5_{c}$ & 13,8 \\
\hline Dominio personal & $81,3_{a, b}$ & 12,0 & $85,4_{a, c}$ & 9,5 & $84,0_{b}$ & 10,8 & $82,2_{c}$ & 11,7 \\
\hline Dominio relacional & $85,6 a$ & 11,7 & $87,9 a$ & 9,4 & 86,6 & 10,7 & 86,0 & 10,7 \\
\hline Dominio ambiental & $88,6 a$ & 8,6 & $90,0_{a, b}$ & 7,8 & 89,0 & 8,7 & $88,4_{b}$ & 8,1 \\
\hline \multicolumn{9}{|l|}{ Mujeres $(n=1.486)$} \\
\hline Índice total & $86,5_{a}$ & 10,0 & $89,5_{a, b}$ & 8,2 & 87,2 & 9,5 & $85,3_{b}$ & 9,4 \\
\hline Dominio general & $90,7 \mathrm{a}$ & 13,2 & $93,9 a, b, c$ & 9,3 & $90,7_{b}$ & 12,8 & $89,4_{c}$ & 12,9 \\
\hline Dominio personal & $81,0_{a, b}$ & 12,1 & $85,3_{a, c}$ & 10,3 & $83,3_{b}$ & 10,7 & $79,7_{c}$ & 12,8 \\
\hline Dominio relacional * & $85,7 \mathrm{a}$ & 12,0 & $88,0_{a}$ & 10,4 & 86,0 & 11,4 & 85,0 & 11,5 \\
\hline Dominio ambiental * & $88,7 \mathrm{a}$ & 8,3 & $90,6 a, b$ & 7,9 & 88,7 & 8,9 & $87,3_{b}$ & 8,6 \\
\hline \multicolumn{9}{|l|}{ Hombres $(n=915)$} \\
\hline Índice total & $86,4 a$ & 10,2 & $89,2_{a}$ & 6,4 & 88,6 & 9,0 & 87,8 & 9,3 \\
\hline Dominio general & $90,2 \mathrm{a}$ & 14,5 & $94,3_{a}$ & 6,9 & 92,8 & 12,1 & 91,3 & 14,4 \\
\hline Dominio personal & $82,0_{a, b}$ & 11,7 & $85,6 a$ & 8,3 & $84,9 b$ & 10,9 & 83,9 & 10,6 \\
\hline Dominio relacional & $85,3 a$ & 10,8 & $87,8 a$ & 7,7 & 87,3 & 19,0 & 86,8 & 10,1 \\
\hline Dominio ambiental & 88,1 & 9,2 & 89,1 & 7,4 & 89,3 & 8,4 & 89,1 & 7,7 \\
\hline
\end{tabular}

DS: desviación estándar.

Nota: la ANOVA de todos los grupos fue significativa con $p<0,01$, excepto para hombres el dominio ambiental. Para todos los grupos la prueba post hoc aplicada fue Tamhane's, excepto para aquellos señalados con *, en los que la prueba post hoc aplicada fue Scheffé. En cada fila las medias que comparten algún subíndice (letras a, b, c, d, e) son significativamente diferentes entre ellas con una $\mathrm{p}<0,05$.

frecuencias son más altas en nuestro estudio que el YRBSS 2007 y algo más bajas que en la ENSANUT, (respectivamente en mujeres 36,5\%, 34,5\%, $37,1 \%$ y en hombres $30,6 \%, 24,2 \%$ y $33,8 \%$ ). Las diferencias con el YRBSS 2007 podrían atribuirse a ser datos de diferentes culturalmente y haber incluido población más joven (14 a 17 años) y con la ENSANUT a que la población estudiada no es una muestra representativa de la población adolescente estatal. Del mismo modo que en la ENSANUT, se encontró en personas de 12 a 19 años que la prevalencia de percepción de sobrepeso fue mayor en poblaciones urbanas que en rurales, en nuestro estudio se encontró significativamente mayor percepción del sobrepeso en procedencia urbana que procedencia campesina, lo que refuerza la idea de la cultura urbana como propiciadora del riesgo, incluyendo el efecto de los medios sobre la imagen corporal.

En nuestro estudio $44,7 \%$ tratan de bajar de peso, prevalencia similar al $45,2 \%$ reportado por el YRBSS 2007 1. La prevalencia en las mujeres que intentan bajar de peso es menor al reportado en el YRBSS 2007 (52,8\% versus 60,3\%), pero en hombres es semejante $(31,7 \%$ versus $30,4 \%)$. Cuando analizamos si este intento de bajar de peso va ligado a una percepción de sobrepeso, encontramos que un $40 \%$ de las mujeres que perciben su peso cercano a lo correcto tratan de bajar de peso Estas diferencias por género ya se habían encontrado en un estudio australiano 17 y coincide con la imagen social de la mujer occidental y la presión social para ser aceptadas 18 .

Hay una prevalencia mayor de comportamientos de control de peso que podemos considerar saludables (hacer ejercicio y comer menos alimentos, menos calorías o comidas bajas en grasa) que los no saludables (dejar de comer, hacer dieta, vomitar o tomar laxantes). Al comparar nuestros resultados con los reportados por el YRBSS $2007{ }^{1}$ para perder o evitar subir de peso, todos los comportamientos de control del peso tuvieron en los nuestros prevalencia menor. Las diferencias podrían deberse al mayor control social en que se desenvuelven los estudiantes investigados. El hecho de que la frecuencia encon- 
Índice total de calidad de vida en población total y por género, según presencia de comportamientos de control del peso en adolescentes mexicanos.

\begin{tabular}{|c|c|c|c|c|c|}
\hline \multirow[t]{3}{*}{ Grupo } & \multicolumn{4}{|c|}{ Comportamientos de control del peso } & \multirow[t]{3}{*}{ Valor-p } \\
\hline & \multirow[b]{2}{*}{ Media } & No & \multicolumn{2}{|c|}{ Sí } & \\
\hline & & DS & Media & DS & \\
\hline \multicolumn{6}{|c|}{ Hacer ejercicio } \\
\hline Total & 86,9 & 9,4 & 88,1 & 9,1 & 0,002 \\
\hline Mujeres & 86,7 & 9,5 & 87,8 & 9,5 & 0,023 \\
\hline Hombres & 87,3 & 9,2 & 88,6 & 8,4 & 0,032 \\
\hline \multicolumn{6}{|c|}{ Comer menos } \\
\hline Total & 88,1 & 8,6 & 86,5 & 10,2 & $<0,001$ \\
\hline Mujeres & 88,0 & 8,6 & 86,3 & 10,5 & $<0,001$ \\
\hline Hombres & 88,2 & 8,7 & 87,2 & 9,3 & 0,142 \\
\hline \multicolumn{6}{|c|}{ Dejar de comer por 24 horas } \\
\hline Total & 87,8 & 8,9 & 79,5 & 13,4 & $<0,001$ \\
\hline Mujeres & 87,6 & 9,2 & 79,9 & 13,3 & $<0,001$ \\
\hline Hombres & 88,2 & 8,5 & 78,6 & 13,9 & $<0,001$ \\
\hline \multicolumn{6}{|c|}{ Dieta sin supervisión } \\
\hline Total & 87,7 & 9,1 & 83,4 & 12,3 & $<0,001$ \\
\hline Mujeres & 87,5 & 9,3 & 82,7 & 13,0 & $<0,001$ \\
\hline Hombres & 88,0 & 8,8 & 86,4 & 8,1 & 0,478 \\
\hline \multicolumn{6}{|c|}{ Vomitar o tomar laxantes } \\
\hline Total & 87,7 & 9,0 & 79,1 & 14,5 & $<0,001$ \\
\hline Mujeres & 87,6 & 9,1 & 77,6 & 14,3 & $<0,001$ \\
\hline Hombres & 88,0 & 8,8 & 84,9 & 14,2 & 0,471 \\
\hline
\end{tabular}

DS: desviación estándar.

Nota: el valor-p fue resultado de una prueba t para muestras independientes.

trada de comportamientos de control del peso no saludables es mayor en mujeres que en hombres, coincide con un estudio en EEUU en 4.746 adolescentes de escuelas públicas, donde la existencia de estos comportamientos fue reportado en un $18 \%$ de las adolescentes y en un $6 \%$ de los adolescentes con mucho sobrepeso 32 .

En cuanto a CV, las puntuaciones tienden hacia valores altos, tanto en las medias como en los puntos de corte observados, lo que refleja para este instrumento que son personas que tienen condiciones favorables al ser admitidos en la universidad y que las puntuaciones más bajas en el dominio personal son, probablemente, debidas a que durante la adolescencia el individuo aún mantiene aspectos de su identidad sin consolidar. El mismo instrumento, cuando fue aplicado con adolescentes norteamericanos 26 , obtuvo las menores puntuaciones en este dominio, mientras el más alto fue el dominio ambiental, igual que en nuestro estudio.

Encontramos la percepción del sobrepeso asociado a la menor $\mathrm{CV}$, y la percepción del peso cercano al correcto a la mejor puntuación, sugiriendo que las desviaciones de la percepción del peso normal afectan la CV. Un grupo de estudios que informan lo mismo en población infantil y adolescente, usando medidas objetivas de peso y que usaron el Pediatric Quality of Life Inventory (Peds QL) 33,34,35,36,5,37, el Peds QL antes de $2008{ }^{8}$ y el Medical Outcomes Study Short Forms 36 (SF-36) 7 y un estudio en adolescentes alemanes que midió peso, percepción de peso y calidad de vida mediante el KINDL, destacando que tienen menor CV que los obesos, quienes se perciben con mucho sobrepeso, y los con peso normal su calidad baja significativamente al percibirse con sobrepeso 38 .

Los adolescentes con mucho sobrepeso presentan la CV más baja, no sólo en el índice total, sino en el dominio general, personal, relacional y ambiental, tanto para la población total como por género, lo cual sugiere que impacta en una extensa área de la vida del adolescente. Coincide con un estudio norteamericano en una muestra de niños de 8-12 años que encontró menor 
puntuación en CV total y dominios psicosocial y físico en obesos que en quienes tenían sobrepeso 36 , también coincide con un estudio realizado en Israel que utilizó el Peds QL y encontró diferencias en $\mathrm{CV}$ en los dominios físico, social y escolar entre extremadamente obesos y peso normal 33, y finalmente con un estudio hecho en EEUU que utilizó el Peds QL con adolescentes méxico-americanos y reportó menor CV física y salud psicosocial en los con sobrepeso, en relación a los de peso normal 37 . En mujeres nuestros resultados coinciden con los de otros investigadores ${ }^{39}$ que encontraron menor $\mathrm{CV}$ en mujeres con ligero sobrepeso, respecto a las de peso normal.

Hallamos menor índice total y dominio personal de CV en los hombres con ligero sobrepeso, respecto a los de peso cercano al correcto, lo que difiere de un estudio hecho en Canadá 39 con 6.302 mujeres y 2.792 hombres mayores de 25 años que encontró que la CV fue ligeramente mejor, incluso para los hombres con ligero sobrepeso, respecto a los de peso normal y de otro estudio que encontró que los hombres con sobrepeso tenían mejores puntuaciones en los dominios mentales que los de peso normal 40 . Probablemente, en esos estudios donde la edad fue mayor en los hombres se observó una menor preocupación por la apariencia personal que en los adolescentes, como en nuestro estudio, donde su apariencia es aún un aspecto importante de su vida interior.

En esta investigación quienes tratan de mantenerse en su peso obtienen las mejores puntuaciones. Esto habla de que están conformes o les parece un peso adecuado. La inconformidad con su peso en quienes quieren subir o bajar o su deseo de alcanzar otro se relacionó con menor calidad de vida, lo que podría reflejar una insatisfacción, aunque hay autores que han encontrado que quienes tienen comportamientos de control saludables como ejercicio o dieta tienen mejor CVRS 9, la diferencia en los instrumentos podría explicar las diferencias por los dominios y preguntas considerados. En nuestro estudio la CV más baja en mujeres que tratan de bajar o subir de peso, que no se presenta en hombres, coincide con el estudio de Clemens et al. 18 quienes reportaron en mujeres mayor preocupación respecto al peso.

En cuanto a comportamientos saludables de control del peso, los que hacen ejercicio, en ambos géneros, tienen mejor $\mathrm{CV}$ que los que no hacen, similar a estudios que encontraron mayor CV en quienes hicieron ejercicio 9,19,20 y también respecto a un estudio norteamericano, hecho con 177 niños de 8 a 12 años con sobrepeso u obesidad, usando el Peds QL que encontró que los que eran menos activos físicamente tenían menor CV psicosocial y total independientemente de su peso 36 . La CV mayor en los que hicieron ejercicio para bajar de peso en nuestro estudio puede estar relacionada con la evidencia de beneficios físicos y psicológicos vinculados a la actividad física y en adolescentes ésta se ha relacionado con un decremento en ansiedad y depresión, mejora en relaciones parentales, incrementada autoestima y menor enojo, estrés psicológico y menores problemas de salud mental 20.

Nuestros hallazgos relativos a la CV menor para los que comen menos alimentos, dejan de comer, hacen dieta y vomitan, comparados con quienes no lo hacen, coinciden con los estudios de Hassan et al. ${ }^{9}$ y se relacionan con estudios hechos en pacientes con trastornos alimentarios 21,22. En el caso de los que comieron menos el último mes y los que no, Haines \& NeumarkSztainer 41 identificaron diversos estudios que asociaron dieta a ganar peso y dieta a desórdenes alimentarios.

En referencia a la conducta de dejar de comer durante 24 horas, que puede ser presentada por personas con desórdenes alimentarios, podría ser similar a lo planteado por Doll et al. 21, quienes encontraron que los sujetos con desórdenes alimentarios reportaron peores puntuaciones en el bienestar físico y emocional al utilizar el SF-36. Del mismo modo, Mond et al. 42 al usar la versión abreviada de The World Health Organization Quality of Life Assessment Instrument (WHOQoLBREF) y el SF-12, entre grupos de pacientes con desórdenes alimentarios, encontraron que tomados juntos mostraban un marcado empeoramiento en lo referente a la CV relacionada a la salud y la CV subjetiva relacionada con el grupo control. Sin embargo, en nuestro estudio no son sujetos quienes tienen un diagnóstico, sino que auto reportan una conducta.

La presencia de comportamientos no saludables ha sido relacionada con problemas de salud física y psicológica ${ }^{2}$ y en nuestro estudio el índice total de CV es más bajo en quienes consumen menos alimento, hacen dieta sin supervisión o vomitan para bajar de peso comparado con quienes no lo hacen. Al separar en grupos por género, las diferencias se mantienen sólo en mujeres, lo que señala el impacto mayor que tiene en ellas este tipo de comportamientos.

Este estudio destaca las áreas o dominios de la CV que están asociados con el sobrepeso y los comportamientos llevados a cabo para su control, esta información puede ser de utilidad para procesos educativos, programas de prevención y para evaluar las intervenciones.

Como limitaciones podemos señalar que el estudio es transversal, las relaciones causales, 
por tanto, no pueden ser determinadas. El tamaño de la población se redujo con algunos sujetos que se excluyeron por no haber contestado el cuestionario completo o porque no asistieron a la aplicación y que pudieran haber tenido características especiales. Algunos participantes podían haber dado respuestas socialmente deseables. Las diferencias en las variables de edad y trabajo entre géneros que no fueron analizadas específicamente podrían explicar algunas diferencias encontradas.

El auto reporte pudo sesgar nuestros resultados por haber subestimado el peso real, como se ha reportado en otros estudios $16,43,44$, por lo que podríamos tener más o menos personas con sobrepeso que las que aparecen. Pero los estudios sobre medidas auto referidas del peso han señalado su utilidad 2 mayor para poblaciones grandes, lo que permite disminuir el costo y tiempo y con población joven, donde la diferencia entre peso reportado son similares a los medidos 43 .

\section{Consideraciones finales}

El estudio de las percepciones y comportamientos de control del peso corporal, y la asociación con la calidad de vida de adolescentes, muestra frecuencias altas de percepción de sobrepeso que generan búsquedas de control no saludables que afectan su calidad de vida, especialmente en mujeres. La medición de las condiciones físicas, y sus respectivas percepciones en población universitaria, resulta indispensable para formular científicamente programas de educación física y alimentación.

\section{Resumen}

El objetivo de este estudio fue analizar la calidad de vida $(\mathrm{CV})$, según auto percepción del peso corporal y comportamientos de control de peso, por género, en 2.401 estudiantes de 17 a 19 años de primer ingreso en un centro universitario en México del 2007 al 2009; $61,9 \%$ mujeres, $80,6 \%$ no trabajaban y 99,2\% solteros. Se auto administró en línea un cuestionario genérico de CV (YQOL-R) módulo perceptual, y siete ítems acerca del peso adaptados del YRBS 2007. Se observó que un 52\% de mujeres y un 31,7\% de hombres trataba de bajar de peso. CV más alta para peso cercano al correcto, quienes trataban de mantenerse en su peso y los que hacían ejercicio; CV más baja para quienes reportaron mucho sobrepeso, mujeres que trataban de bajar de peso, comían menos, dejaban de comer, hacían dieta sin supervisión, vomitaban o tomaban laxantes. En mujeres la CV fue diferente si trataban de mantenerse, subir o bajar de peso; en hombres sólo al tratar de subir. Esta información puede ser de utilidad para procesos educativos, programas de prevención y para evaluar las intervenciones.

\section{Colaboradores}

C. A. Hidalgo-Rasmussen contribuyó con la concepción del estudio, adquisición, análisis e interpretación de los datos, redacción del artículo y aprobación de la versión final. A. Hidalgo-San Martín contribuyó con la concepción del estudio, diseño del estudio, interpretación de los datos, revisión crítica y aprobación de la versión final. B. Rasmussen-Cruz contribuyó con la interpretación de los datos, redacción del artículo, revisión crítica del artículo y aprobación de la versión final. R. MontañoEspinoza contribuyó con el diseño del estudio, análisis e interpretación de los datos, revisión crítica del artículo y aprobación de la versión final.

\section{Agradecimientos}

Se agradece al apoyo del Centro Universitario del Sur de la Universidad de Guadalajara para llevar a cabo este estudio y al Mtro. Gerardo Jiménez-Haro por su apoyo técnico en la programación de la base de datos del cuestionario en línea. 


\section{Referencias}

1. Eaton DK, Kann L, Kinchen S, Shanklin S, Ross J, Hawkins J, et al. Youth risk behavior surveillance United States, 2007. MMWR Surveill Summ 2008; 57:1-131.

2. Centers for Disease Control and Prevention. YRBSS: Youth Risk Behavior Surveillance System. http://www.cdc.gov/HealthyYouth/yrbs/pdf/ questionnaire/2009ItemRationale.pdf (accedido el 14/Feb/2010).

3. Shamah-Levy T, Villalpando-Hernández S, RiveraDommarco JA. Resultados de nutrición de la ENSANUT 2006. Cuernavaca: Instituto Nacional de Salud Pública; 2007.

4. Olaiz-Fernández G, Rivera-Dommarco J, ShamahLevy T, Rojas R, Villalpando-Hernández S, Hernández-Avila M. Encuesta Nacional de Salud y Nutrición 2006. 2a Ed. Cuernavaca: Instituto Nacional de Salud Pública; 2006.

5. Swallen K, Reither M, Haas S, Meier A. Overweight, obesity, and health-related quality of life among adolescents: the National Longitudinal Study of Adolescent Health. Pediatrics 2005; 115:340-7.

6. Kunkel N, Ferreira W, Aurélio M. Overweight and health-related quality of life in adolescents of Florianópolis, Southern Brazil. Rev Saúde Pública 2009; 43:1-9.

7. Larsson U, Karlsson J, Sullivan M. Impact of overweight and obesity on health-related quality of life - a Swedish population study. Int J Obes 2002; 26:417-24.

8. Tsiros MD, Olds T, Buckley JD, Grimshaw P, Brennan L, Walkley J, et al. Health-related quality of life in obese children and adolescents. Int J Obes 2009; 33:387-400

9. Hassan MK, Joshi AV, Madhavan SS, Amonkar MM. Obesity and health-related quality of life: a crosssectional analysis of the US population. Int J Obes 2003; 27:1227-32.

10. The WHOQoL Group. Study protocol for the World Health Organization project to develop a quality of life assessment instrument (WHOQoL). Qual Life Res 1993; 2:153-9.

11. The WHOQoL Group. The World Health Organization Quality of Life Assessment (WHOQoL): position paper from the World Health Organization. Soc Sci Med 1995; 41:1403-9.

12. Cummins R, Cahill J. Avances en la comprensión de la calidad de vida subjetiva. Interv Psicosoc 2000; 9:185-98.

13. Cummins R. Moving from the quality of life concept to a theory. J Intellec Disabil Res 2005; 10: 699-706.

14. Rajmil L, Estrada MD, Herdman M, Serra-Sutton V. Calidad de vida relacionada con la salud (CVRS) en la infancia y la adolescencia: revisión de la bibliografía y de los instrumentos adaptados en España. Gac Sanit 2001; 15:34-43.

15. Guyatt G, Feeny D, Patrick D. Measuring healthrelated quality of life. Ann Intern Med 1993; 118:622-9.

16. Brener ND, Kann L, Kinchen SA, Grunbaum JA, Whalen L, Eaton D, et al. Methodology of the youth risk behavior surveillance system. MMWR Recomm Rep 2004; 53(RR-12):1-13.
17. O’Dea J, Caputi P. Association between socioeconomic status,weight, age and gender, and the body image and weight control practices of 6- to 19-year-old children and adolescents. Health Educ Res 2001; 16:521-32.

18. Clemens H, Thoms D, Olds S, Lowry K. Normative beliefs as risk factors for involvement in unhealthy weight control behavior. J Am Coll Health 2008; 56:635-41.

19. Vaez M, Laflamme L. Health behaviors, self-rated health, and quality of life: a study among first year Swedish University students. J Am Coll Health 2003; 51:156-62.

20. Valois R, Zullig K, Huebner E, Dane W. Physical activity behaviors and perceived life satisfaction among public high school adolescents. J Sch Health 2004; 74:59-65.

21. Doll H, Petersen S, Stewart-Brown S. Eating disorders and emotional and physical well-being: associations between student self-reports of eating disorders and quality of life as measured by the SF36. Qual Life Res 2005; 14:705-17.

22. De La Rie S, Noordenbosw G, Van Furth E. Quality of life and eating disorders. Qual Life Res 2005; 14:1511-22.

23. Topolski T, Edwards T, Patrick D. User's manual and interpretacion guide for the Youth Quality of Life (YQOL) instruments. Seattle: Department of Health Services, University of Washington; 2002.

24. Patrick D, Edwards T, Topolski T, Walwick J. Youth quality of life: a new measure incorporating the voices of adolescents. Quality of Life Newsletter 2002; 28:7-8.

25. Wee H-L, Ravens-Sieberer U, Erhart M, Li S-C. Factor structure of the Singapore English version of the $\mathrm{KINDL}^{\circledR}$ children quality of life questionnaire. Health Qual Life Outcomes 2007; 5:1-8.

26. Patrick D, Edwards T, Topolski T. Adolescent quality of life. Part II: initial validation of a new instrument. J Adolesc Health 2002; 25:287-300.

27. Chávez L, Matías-Carrelo L, Barrio C, Canino G. The cultural adaptation of the Youth Quality of Life Instrument-Research version for Latino children and adolescents. J Child Fam Stud 2007; 16:72-86.

28. Scientific Advisory Comitee of the Medical Outcomes Trust. Assessing health status and qualityof-life instruments: attributes and review criteria. Qual Life Res 2002; 11:193-205.

29. Centers for Disease Control and Prevention. State and local Youth Risk Behavior Survey. http://www.cdc.gov/healthyyouth/yrbs/pdf/ questionnaire/2007HighSchool.pdf (accedido el 20/Jul/2006)

30. Katchadourian H. La sexualidad humana. Un estudio comparativo de su evolución. México DF: Fondo de Cultura Económica; 1983.

31. Instituto Nacional de Salud Pública. Encuesta Nacional de Salud y Nutrición 2006. Resultados por entidad federativa. Cuernavaca: Instituto Nacional de Salud Pública; 2007.

32. Neumark-Sztainer D, Story M, Hannan PJ, Perry CL, Irving LM. Weight-related concerns and behaviors among overweight and nonoverweight adolescents. Arch Pediatr Adolesc Med 2002; 156:171-8. 
33. Pinhas-Hamiel O, Singer S, Pilpel N, Fradkin A, Modan D, Reichman B. Health-related quality of life among children and adolescents: associations with obesity. Int J Obes 2006; 30:267-72.

34. Zeller M, Modi A. Predictors of helath-related quality of life in obese youth. Obesity 2006; 14:122-30.

35. Janicke DM, Marciel KK, Ingerski LM, Novoa W, Lowry KW, Sallinene BJ, et al. Impact of psychosocial factors on quality of life in overweight youth. Obesity 2007; 15:1977-807.

36. Shoup JA, Gattshall M, Dandamudi P, Estabrooks P. Physical activity, quality of life, and weight status in overweight children. Qual Life Res 2008; 17: 407-12.

37. Tyler C, Johnston C, Fullerton G, Foreyt JP. Reduced quality of life in very overweight Mexican American adolescents. J Adolesc Health 2007; 40:366-8.

38. Kurth BM, Ellert U. Perceived or true obesity: which causes more suffering in adolescents? Findings of the German Health Interview and Examination Survey for Children and Adolescents (KiGGS) Dtsch Arztebl Int 2008; 105:406-12.
39. Hopman WM, Berger C, Joseph L, Barr SI, Gao Y, Prior JC, et al. The association between body mass index and health-related quality of life: data from CaMos, a stratified population study. Qual Life Res 2007; 16:1595-603.

40. Huang IC, Frangakis C, Wu AW. The relationship of excess body weight and health-related quality of life: evidence from a population study in Taiwan. Int J Obes 2006; 30:1250-9.

41. Haines J, Neumark-Sztainer D. Prevention of obesity and eating disorders: a consideration of shared risk factors. Health Educ Res 2006; 21:770-82.

42. Mond J, Hay P, Rodgers B, Owen C, Beumont P. Assessing quality of life in eating disorder patients. Qual Life Res 2005; 14:171-8.

43. Kuczmarski MF, Kuczmarski MRJ, Najja M. Effects of age on validity of self-reported height, weight, and body mass index: findings from the third $\mathrm{Na}-$ tional Health and Nutrition Examination Survey, 1988-1994. J Am Diet Assoc 2001; 101:28-34.

44. Brunner-Huber LR. Validity of self-reported height and weight in women of reproductive age. Matern Child Health J 2007; 11:137-44.

Recibido el 18/Abr/2010

Versión final presentada el 02/Sep/2010

Aprobado el 21/Sep/2010 